\title{
Commentary: Food intake regulation by leptin: Mechanisms mediating gluconeogenesis and energy expenditure
}

'Institute of Pharmacy, Physiology and Pharmacology, University of Agriculture Faisalabad (UAF)-38040, Pakistan.

Article Info

\section{Article Notes}

Received: January 30, 2018

Accepted: February 16, 2018

\section{*Correspondence:}

Dr. Junaid Ali Khan, Assistant Professor, Institute of Pharmacy, Physiology and Pharmacology, University of Agriculture, Faisalabad (UAF), Pakistan;

Telephone: +92 41 9200161-70;

Fax: +92 41 9200764;

E-mail: junaidali.khan@uaf.edu.pk

(c) 2018 Khan JA. This article is distributed under the terms of the Creative Commons Attribution 4.0 International License.
Leptin is a peptide hormone mainly produced by adipose tissues and involved in the regulation of energy balance, metabolism, and appetite. Presently, it has been recognized as one of the therapeutic candidates for diabetes, hypertriglyceridemia, to regulate appetite and bone mass (first recognized in 2000) and to improve cognitive performance ${ }^{1}$. Studies on transgenic mouse models of Alzheimer's disease have shown that chronic administration of leptin can alleviate brain pathology and improve cognitive function by suppressing $\beta$-amyloid and hyperphosphorylation of tau protein, the hallmarks of Alzheimer's pathology ${ }^{2}$. It was approved for the first time in the US in 2014 for alleviating lipodystrophy and congenital leptin deficiency.

In a generous view, leptin is a naturally occurring anorexigenic hormone, the action of which is paradoxical to the hunger hormone ghrelin, hence decreases food intake. It plays a crucial role in body weight regulation by acting on the hypothalamic receptors of the central nervous system to suppress appetite and burn fat stored in adipose tissue. A number of clinical investigations show that its levels are much higher between midnight and early morning and may be modified by meal-timing. It helps glucose regulation by two distinct ways, i.e., 1) Energy balance (food intake and expenditure), 2) direct action on tissues/genes. It can cross the blood brain barrier and have direct actions on the leptin receptors (ob-rb type) present in the arcuate nucleus of the hypothalamus. These receptors signal intracellularly via activating the Janus kinase-signal transducer and activator of transcription (JAK-STAT) and mitogen-activated protein kinase (MAPK) pathway that regulate transcription of numerous proteins like insulin-like growth factor (IGF-1). The JAKSTAT signaling pathway transmits information from extracellular chemical signals to the nucleus, resulting in DNA transcription and expression of genes therefore regulate appetite in the achievement of energy balance ${ }^{3}$. These receptor isoforms are widely distributed in many organs include liver, pancreas, kidney, heart and adipose tissue $^{4}$. Leptin regulates energy expenditures and food intake on top of glucose homeostasis and hepatic insulin sensitivity suggesting the necessity of maintaining the levels in blood, liver and adipose tissues within narrow range $e^{5}$. The major action of leptin in hepatocytes is to increase glycogen storage and to suppress gluconeogenesis ${ }^{6}$. Increased circulating leptin increases energy expenditure and decreases food intake ${ }^{7}$. In this commentary, we have mainly focused the effects of leptin with special application in gluconeogenesis, 
suggesting different factors that influence leptin secretion and associate energy expenditure and obesity.

Plasma leptin level is closely associated with the percent body fat proposing that leptin is an important factor for maintenance of fat storage and weight regulation. A number of scientific studies on human and rodents propose that there is a complex system of leptin mechanism and other cytokines integrating and coordinating the information to several regions of hypothalamus to regulate energy balance and appetite. Any defects in leptin signaling or hyperleptinemia, resistance to leptin often occurs that can lead to weight gain and obesity. The occurrence of obesity is increasing in many countries. According to global health survey 2014 , about 2.1 billion people were categorized as overweight or obese in the world, and about 2.8 million people die each year due to obesity. In Pakistan, it is one third of the adult population and ranks $9^{\text {th }}$ in the list of countries with the most obese population ${ }^{8}$.

The presence of obesity is a significant risk factor in the progression of hepatic steatosis and fibrosis as it is found that obesity is the predominant cause of nonalcoholic hepatic steatosis. More than two-thirds of patients with nonalcoholic fatty liver disease (NAFLD) are obese 9 . In western countries, NAFLD is the most common cause of chronic liver disease, and it is estimated that it will be the most common cause of liver transplantation by $2030^{10}$. Hepatic steatosis is characterized by the accumulation of fat particles in the liver and inflammation outcomes fibrosis (nonalcoholic steatohepatitis, NASH) ${ }^{11}$. Chronic elevation of free fatty acid levels in the body affects protein levels and gene expression of enzymes intricate in hepatic glucose and lipid metabolism however the molecular mechanisms affect the physiology of glucose and lipid metabolism in the liver is still unclear. Chronic administration of leptin improves glucose tolerance by decreasing fat mass and caloric intake and inhibits hepatic lipogenesis and stimulates free fatty acids oxidation. As in general, the decrease in hepatic glucose production decreases hepatic triglyceride content, hence, suppresses hepatic glycogenolysis and gluconeogenesis. In this way, leptin also has anti-steatotic action.

Recent literature indicates that specific modulation of leptin receptors and adipose tissues functioning could have an important implication on therapeutic strategies $^{3}$. Although many new drugs have only been tested on animals, the findings have implications for the development of new treatments not only for obesity but liver ailments too in humans ${ }^{12}$. We can now suggest that in addition to therapy of obesity new doors are open to utilize the beneficial effects of leptin in liver disorders keeping in view the mechanistic effects on gluconeogenesis and glucose utilization in energy balance through liver. In conclusion, leptin is not only important for regulation of fat consumption and energy expenditure in the body but also has beneficial effects on steatosis though the clinical data on leptin levels in NASH-related cirrhosis or hepatocellular carcinoma is scarce, considerable work is needed to redefine the nature and magnitude of leptin's activities in the liver and GIT.

\section{Conflict of interests}

The authors declare that they have no conflict of interest.

\section{Funding Authority}

Higher Education Commission, Islamabad, Pakistan. ZH is recipient of HEC Indigenous PhD Scholarship from Higher Education Commission, Islamabad, Pakistan (Tracking Id: 213-58222-2BM2-162).

\section{References}

1. Ducy $\mathrm{P}$, Amling $M$, Takeda $\mathrm{S}$, et al. Leptin inhibits bone formation through a hypothalamic relay: a central control of bone mass. Cell. 2000; 100 (2): 197-207.

2. Femminella GD, Bencivenga L, Petraglia L, et al. Antidiabetic Drugs in Alzheimer's Disease: Mechanisms of Action and Future Perspectives. J Diabetes Res. 2017.

3. Hussain Z, Khan JA. Food intake regulation by leptin: Mechanisms mediating gluconeogenesis and energy expenditure. Asian Pac J Trop Med. 2017; 10(10): 940-4.

4. Amitani M, Asakawa A, Amitani $\mathrm{H}$, et al. The role of leptin in the control of insulin-glucose axis. Front Neurosci. 2013; 7: 51.

5. Denroche HC, Huynh FK, Kieffer TJ. The role of leptin in glucose homeostasis. J Diabetes Investig. 2012; 3(2): 115-29.

6. Liu RH, Mizuta M, Matsukura S. Long-term oral nicotine administration reduces insulin resistance in obese rats. Eur J Pharmacol. 2003; 458(1-2): 227-34.

7. Sharma M, Mohapatra J, Malik U, et al. Effect of pioglitazone on metabolic features in endotoxemia model in obese diabetic $\mathrm{db} / \mathrm{db}$ mice. J Diabetes. 2017; 9(6): 613-21.

8. Pakistan 9th most obese country: Express Trib. 2014; Available from: https://tribune.com.pk/story/714845/pakistan-9th-most-obesecountry-study/.

9. Jung UJ, Choi MS. Obesity and its metabolic complications: the role of adipokines and the relationship between obesity, inflammation, insulin resistance, dyslipidemia and nonalcoholic fatty liver disease. Int J Mol Sci. 2014; 15(4): 6184-223.

10. Byrne CD, Targher G. NAFLD: a multisystem disease. J Hepatol 2015; 62(1 Suppl): S47-64.

11. Ghani MU, Ahmad I, Ahmad N, et al. Hematology, Serum Total Cholesterol and Thyroid Hormone Concentrations in Cyclic and Acyclic Nili-Ravi Buffaloes. Pak Vet J. 2017; 37(1): 31-34.

12. Chorvat RJ. Peripherally restricted CB1 receptor blockers. Bioorg Med Chem Lett. 2013; 23(17): 4751-60. 